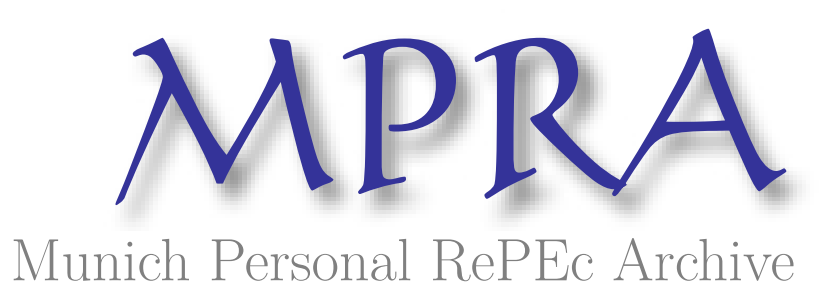

\title{
A Note on the Desirability of the Supply Function Competition with Demand Uncertainty
}

Saglam, Ismail

Ankara, Turkey

28 November 2017

Online at https://mpra.ub.uni-muenchen.de/82995/

MPRA Paper No. 82995, posted 29 Nov 2017 14:29 UTC 


\title{
A Note on the Desirability of the Supply Function Competition with Demand Uncertainty
}

\author{
Ismail Saglam \\ Ankara, Turkey
}

\begin{abstract}
In this paper, we formalize a prediction of Klemperer and Meyer (1989) as to the possibility that in the presence of demand uncertainty the expected profits under the supply function competition may result in higher expected oligopoly profits than under the stochastic Cournot competition and investigate how this possibility is affected by certain attributes of the oligopolistic industry, such as the number of firms, the cost of producing a unit output, and the slope of the demand curve.
\end{abstract}

Keywords: Supply function competition; oligopoly; uncertainty; Cournot competition

JEL Codes: D43; L13

\section{Introduction}

Economists have long criticized Cournot's (1838) oligopolistic competition model for not allowing firms the flexibility to adjust their output in the face of unanticipated demand shocks. A more sophisticated model was proposed by Grossman (1981), according to whom firms can commit to -instead of fixed quantities of outputs- supply functions specifying the quantity to be supplied as a function of the price. However, Grossman (1981) also showed that -in the absence of any type of uncertainties- oligopolistic games with supply function competition may result in a huge multiplicity of pure-strategy equilibria with respect to the noncooperative solution of Nash (1950). Even a more annoying fact is that in the absence of any uncertainties, the competition in supply functions might 
become meaningless in the first place, since all equilibria of supply functions always yield, for each firm, the same profit maximizing quantity of supply (and price), which could have been directly chosen without using any supply function and without incurring any costs due to building a sufficient capacity to credibly commit to a supply function.

However, these arguments against the supply function competition may disappear under some additional assumptions about the demand or cost curves or under some changes in the equilibrium concept, as was shown by Klemperer and Meyer (1989), Delgado and Moreno (2004), Delgado (2006), and Król (2017). The modification devised by Klemperer and Meyer (1989) was to introduce -to the oligopolistic industry- an exogenous demand uncertainty with an unbounded support. In their model, firms simultaneously choose supply functions without knowing the realization of the demand shock/uncertainty. Right after they learn the realization of the uncertainty (hence, the actual position of the demand curve) begins the production stage, where firms can calculate -using the supply functions they chose in the previous stage- a market clearing price and their actual supplies implied by this price. Since each possible realization of the demand curve implies a distinct profit maximizing quantity of supply, the ability of firms to commit, through supply functions, to all possible realizations of the profit maximizing quantities (and all possible market clearing prices) can protect them against the uncertainties they might face. Also, the number (or the measure) of the supply function equilibria would be significantly reduced in the presence of demand uncertainty, because the supply function of each firm would need to pass through not a single but a multiplicity of profit maximizing quantities, each of which corresponding to a distinct realization of the demand curve. In fact, the uncertainty in demand can even ensure the uniqueness of equilibrium if the demand curve and the marginal cost curve of each firm become linear when the price and the supply become sufficiently large.

More recently, Delgado and Moreno (2004) and Delgado (2006), who followed a different path from that of Klemperer and Meyer (1989), realized that if the Nash equilibrium concept is changed with the coalition-proof equilibrium and if some additional conditions hold, not only the uniqueness of the supply function equilibria can be ensured in a deterministic oligopoly but also this unique equilibrium and the Cournot competition can lead to the same outcome. On the other hand, Król (2017) formalized an insight of Klemperer and Meyer (1989) to show that the enormous multiplicity of the supply function equilibria can be reduced in the absence of any uncertainties as well if different supply functions have different costs of implementation. Specifically, Król (2017) showed that 
when excess capacity is always costly, there is even a one-to-one correspondence between the sets of Nash equilibria, and also (under some additional conditions about the industry) between the sets of strategies surviving the iterated elimination of weakly dominated strategies.

An important result of Klemperer and Meyer (1989), distinguishing it from the subsequent works discussed above, is that the set of supply function equilibria under the demand uncertainty can never boil down to the Cournot equilibria of a non-stochastic industry. As a matter of fact, Klemperer and Meyer (1989) showed that for any realization of the demand uncertainty, the equilibrium quantities of supply become always higher, and consequently the equilibrium profits become always lower, under the supply function competition with demand uncertainty than under the Cournot competition without uncertainties. However, they also predicted that when an uncertain demand curve is linear in price and resultingly the supply function competition leads to a unique equilibrium, the expected profits at this equilibrium may be higher than the expected profits obtained under the stochastic Cournot competition where firms choose their supply quantities before observing the realization of the demand uncertainty. The reason they offer is that only under the supply function competition do firms adjust optimally to every possible realization of the demand uncertainty. In this paper, we formalize this prediction of Klemperer and Meyer (1989) as to the possibility of the supply function competition with demand uncertainty resulting in higher expected oligopoly profits than obtained under the stochastic Cournot competition and investigate how this possibility is affected by certain attributes of the oligopolistic industry, involving the number of firms, the cost of producing a unit output, and the slope of the demand curve.

The rest of the paper is organized as follows: Section 2 introduces a simple model of an oligopolistic industry with demand uncertainty. Sections 3 and 4 present for this model the stochastic versions of the supply function competition and the Cournot competition respectively. Section 5 contains our results and finally Section 6 concludes.

\section{Model}

Borrowing from Klemperer and Meyer (1989), we consider an oligopolistic industry involving $n \geq 2$ firms who produce a single homogenous good. The firms have identical cost functions such that each firm producing a quantity of output 
$q$ incurs the cost

$$
C(q)=c q^{2} / 2 \text { for all } q \geq 0,
$$

where $c>0$. The industry demand curve is given by

$$
D(p, \epsilon)=-m p+\epsilon,
$$

where $p \geq 0$ is the market price of the good, $m>0$ and $\epsilon \in[0, \infty)$. The form of the cost and demand curves, $C(q)$ and $D(p, \epsilon)$, as well as the cost and demand parameters $c$ and $m$ are assumed to be commonly known by the firms. On the other hand, $\epsilon$ is a scalar random variable with a probability density $f(\epsilon)$ that is strictly positive everywhere on the support $[0, \infty)$. It is also assumed that there is common knowledge about $f($.$) .$

\section{Supply Function Competition with Demand Uncertainty}

A strategy for firm $i$ is a function mapping price into a quantity of output for this firm, i.e., $S^{i}:[0, \infty) \rightarrow(-\infty, \infty)$. In the pre-production stage, firms simultaneously choose supply functions without knowing the realization of the demand variable $\epsilon$. Right after they learn the realization of $\epsilon$ begins the production stage, where firms calculate -using the supply functions they chose in the previous stage- a market clearing price $\bar{p}(\epsilon)$ that satisfies

$$
\sum_{i=1}^{n} S^{i}(\bar{p}(\epsilon))=D(\bar{p}(\epsilon), \epsilon)
$$

If this price exists and if it is unique, then the actual outputs $\left(S^{i}(\bar{p}(\epsilon))\right)_{i=1}^{n}$ are produced. Otherwise, each firm earns zero profits.

For the game played in the first stage of the above setup we focus on the Nash equilibria in supply functions as in Grossman (1981) and Klemperer and Meyer (1989). We say that a profile (list) of supply functions $\left(\bar{S}^{i}(p)\right)_{i=1}^{n}$ is a Nash equilibrium if for each firm $i$ the function $\bar{S}^{i}(p)$ maximizes its expected

profits when all of the remaining firms stick to their supply functions in the considered profile. That is, for each $i$ the function $\bar{S}^{i}(p)$ solves

$$
\max _{S(p)} E\left[p\left(D(p, \epsilon)-\sum_{j \neq i} \bar{S}^{j}(p)\right)-C\left(D(p, \epsilon)-\sum_{j \neq i} \bar{S}^{j}(p)\right)\right],
$$


where $E$ denotes the expectations operator under the probability density function $f(\epsilon)$ over $\epsilon \in[0, \infty)$.

Proposition 1 (Klemperer and Meyer, 1989). For the model described in Section 2, the supply function competition with demand uncertainty has a unique Nash equilibrium profile of supply functions characterized by

$$
\bar{S}^{i}(p)=\alpha p, \text { for all } i,
$$

where

$$
\alpha=\frac{-m+\frac{n-2}{c}+\sqrt{\left(-m+\frac{n-2}{c}\right)^{2}+\frac{4 m(n-1)}{c}}}{2(n-1)} .
$$

Proof. See the proof of Proposition 8a in Klemperer and Meyer (1989).

Using the equilibrium supply functions characterized by (5) and (6), the demand function in (2), and the market clearing condition in (3), we can calculate for any realization of $\epsilon$ the corresponding market clearing price and the equilibrium quantities as

$$
\bar{p}(\epsilon)=\frac{\epsilon}{n \alpha+m}
$$

and

$$
\bar{S}^{i}(\bar{p}(\epsilon))=\bar{q}(\epsilon)=\frac{\alpha \epsilon}{n \alpha+m} \text { for all } i .
$$

It follows that for any realization of $\epsilon$, the 'realized' profits of each firm will be identical and equal to

$$
\bar{\pi}(\epsilon)=\bar{p}(\epsilon) \bar{q}(\epsilon)-c \bar{q}(\epsilon)^{2} / 2=\frac{\alpha \epsilon^{2}}{(n \alpha+m)^{2}}\left(1-\frac{c \alpha}{2}\right) .
$$

On the other hand, before the firms learn the realization of the demand uncertainty, the equilibrium profit they expect to earn can be calculated by applying the expectations operator $E[$.$] to both sides of the above equation, yielding$

$$
E[\bar{\pi}(\epsilon)]=\frac{\alpha}{(n \alpha+m)^{2}}\left(1-\frac{c \alpha}{2}\right) E\left[\epsilon^{2}\right]
$$




\section{Cournot Competition with Demand Uncer- tainty}

A strategy for firm $i$ is a nonnegative quantity of output, $q^{i} \in[0, \infty)$. So, let us write the expected profits of firm $i$ as a function of $q^{i}$. Let $Q$ denote the industry output; i.e., $Q=\sum_{i=1}^{n} q^{i}$. Inverting (2), we obtain the inverse demand function $P(Q, \epsilon) \equiv D^{-1}(Q, \epsilon)$ as follows:

$$
P(Q, \epsilon)=\frac{\epsilon}{m}-\frac{Q}{m}
$$

for any $Q \geq 0$. Using this, we can write the expected profits of firm $i$ as follows:

$$
E\left[P\left(q^{i}+\sum_{j \neq i} q^{j}, \epsilon\right) q^{i}-C\left(q^{i}\right)\right]
$$

Firms are assumed to simultaneously choose and implement their supplies without knowing the realization of the uncertain demand variable $\epsilon$. In this game we focus on the (Cournot) Nash equilibria in quantities. We say that a profile of quantities $\left(\hat{q}^{i}\right)_{i=1}^{n}$ is a Nash equilibrium if for each firm $i$ the quantity $\hat{q}^{i}$ maximizes its expected profits when all of the remaining firms stick to their quantities in the considered profile. That is, for each $i$ the quantity $\hat{q}^{i}$ solves

$$
\max _{q} E\left[P\left(q^{i}+\sum_{j \neq i} \hat{q}^{j}, \epsilon\right) q^{i}-C\left(q^{i}\right)\right] .
$$

Proposition 2. For the model described in Section 2, the Cournot competition with demand uncertainty has a unique Nash equilibrium profile of quantities characterized by

$$
\hat{q}^{i}=\frac{E[\epsilon]}{n+1+m c} \text { for all } i
$$

Proof. Inserting (1) and (11) into (12), we can rewrite the expected profits of firm $i$ as

$$
E\left[\pi^{i}(\epsilon)\right]=\frac{E[\epsilon]}{m}-\frac{1}{m}\left(q^{i}+\sum_{j \neq i} q^{j}\right) q^{i}-\frac{c}{2}\left(q^{i}\right)^{2} .
$$

Differentiating (15) with respect to $q^{i}$ we obtain the first-order necessary condition

$$
\frac{E[\epsilon]}{m}-\frac{1}{m}\left(2 q^{i}+\sum_{j \neq i} q^{j}\right)-c q^{i}=0,
$$


which implies the following best-response (reaction) function for firm i:

$$
q^{i}=\frac{1}{2+m c}\left(E[\epsilon]-\sum_{j \neq i} q^{j}\right)
$$

Since the reaction functions of the firms are symmetric, we must have $\hat{q}^{j}=\hat{q}^{i}$ for all $j \neq i$ in equilibrium. Inserting this into (17) we obtain

$$
\hat{q}^{i}=\frac{1}{2+m c}\left(E[\epsilon]-(n-1) \hat{q}^{i}\right) .
$$

Solving for $\hat{q}^{i}$ yields equation (14). Finally, we calculate the second-order differential of (15) with respect to $q^{i}$ to obtain

$$
\frac{\partial^{2} E\left[\pi^{i}(\epsilon)\right]}{\partial\left(q^{i}\right)^{2}}=-\frac{2}{m}-c
$$

which is always negative. Thus, the second-order sufficiency condition is also satisfied, implying that the profile of quantities $\left(\hat{q}^{i}\right)_{i=1}^{n}$ satisfying (14) solves the maximization problem of each firm, constituting a Nash equilibrium.

Let $\hat{q}=E[\epsilon] /(n+1+m c)$. From (14), $\hat{q}^{i}=\hat{q}$ for all $i$. So, the equilibrium output of the industry must be equal to

$$
\hat{Q}=n \hat{q}=\frac{n E[\epsilon]}{n+1+m c} .
$$

Using (11) and (20), we can then calculate for any realization of $\epsilon$ the corresponding market clearing price:

$$
\hat{p}(\epsilon)=\frac{1}{m}\left(\epsilon-\frac{n E[\epsilon]}{n+1+m c}\right)
$$

It follows that for any realization of $\epsilon$ the realized profits of each firm are equal to

$$
\hat{\pi}(\epsilon)=\hat{p}(\epsilon) \hat{q}-c \hat{q}^{2} / 2=\frac{\epsilon}{m} \frac{E[\epsilon]}{n+1+m c}-\frac{n / m+c / 2}{(n+1+m c)^{2}}(E[\epsilon])^{2} .
$$

It is easy to check that the expected profits of each firm will then be equal to

$$
E[\hat{\pi}(\epsilon)]=\frac{2+m c}{2 m} \frac{(E[\epsilon])^{2}}{(n+1+m c)^{2}} .
$$




\section{$5 \quad$ Results}

Below, we will investigate the possibility that the supply function competition with demand uncertainty may be ex-ante more desirable than the stochastic Cournot competition. To this aim, we calculate a threshold for the demand uncertainty above which the supply competition always becomes superior to the stochastic Cournot competition with respect to the expected equilibrium profits. First note that

$$
E\left[\epsilon^{2}\right]=(E[\epsilon])^{2}\left[1+\left(\frac{\sigma(\epsilon)}{E[\epsilon]}\right)^{2}\right],
$$

where $\sigma(\epsilon)$ denotes the standard deviation of $\epsilon$ under the density $f(\epsilon)$. In the above equation, the ratio $\sigma(\epsilon) / E[\epsilon]$ is known as the coefficient of variation, which is a unitless measure of relative variability. Moreover, it is independent of the realization of $\epsilon$. Let us denote this ratio by $\mathcal{C V}$. Then, (24) can be rewritten as

$$
E\left[\epsilon^{2}\right]=(E[\epsilon])^{2}\left[1+\mathcal{C} \mathcal{V}^{2}\right] .
$$

Now, using (25) we can rewrite equation (10), which identifies the expected equilibrium profits of each firm under the supply function competition with demand uncertainty:

$$
E[\bar{\pi}(\epsilon)]=\frac{\alpha}{(n \alpha+m)^{2}}\left(1-\frac{c \alpha}{2}\right)\left(1+\mathcal{C} \mathcal{V}^{2}\right)(E[\epsilon])^{2} .
$$

Let us denote by $\mathcal{C V}^{*}$ the value of the coefficient of variation at which the expected equilibrium profits obtained -under demand uncertainty- in the Cournot competition and in the supply function competition become equal. By equating equations (23) and (26), this value can be calculated as:

$$
\mathcal{C V}^{*}=\sqrt{\frac{(2+m c)(n \alpha+m)^{2}}{\alpha m(2-c \alpha)(n+1+m c)^{2}}-1},
$$

where $\alpha$ satisfies (6).

When the coefficient of variation in demand, $\mathcal{C}$, is above (below) the threshold value $\mathcal{C V}^{*}$, the supply function competition leads to higher (lower) expected

profits for each firm than the stochastic Cournot competition. Also, as it should be apparent from (27) along with (6), the threshold $\mathcal{C V}^{*}$ depends on various attributes of the industry structure, involving the number of firms, $n$, the slope of the demand curve, $m$, and the cost of producing a unit output, $c$. Below, we will explore how these attributes affect $\mathcal{C} \mathcal{V}^{*}$. However, due to the complex analytical form of $\mathcal{C V}^{*}(c, m, n)$, characterized by equation (27) along with (6), we conduct our comparative statics analysis with the help of a computer graphics program. 
(i) $\mathrm{m}=1 / 81$

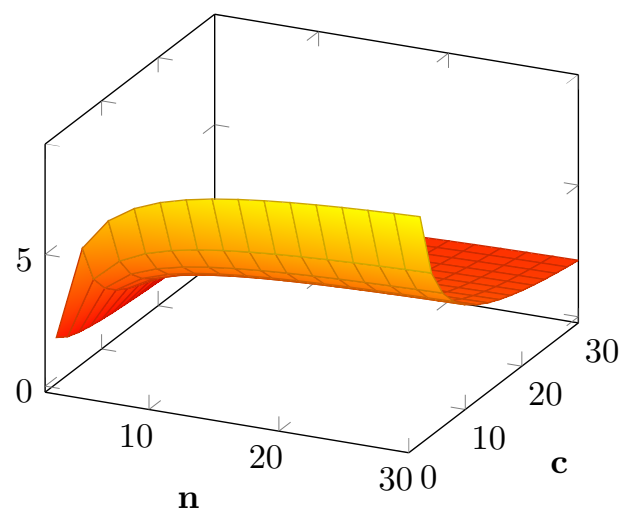

(iii) $\mathrm{m}=1 / 9$

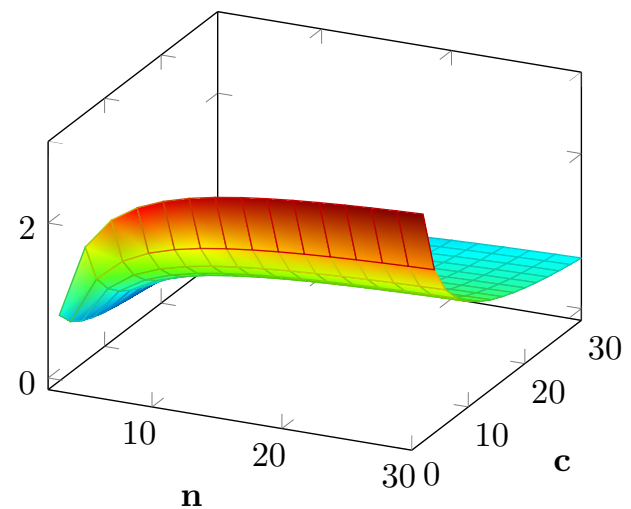

$\mathbf{n}$

(v) $\mathrm{m}=1$

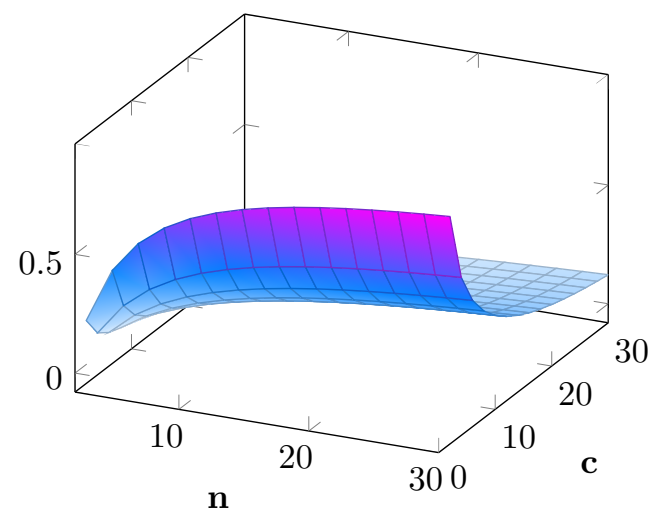

(ii) $\mathrm{m}=1 / 27$

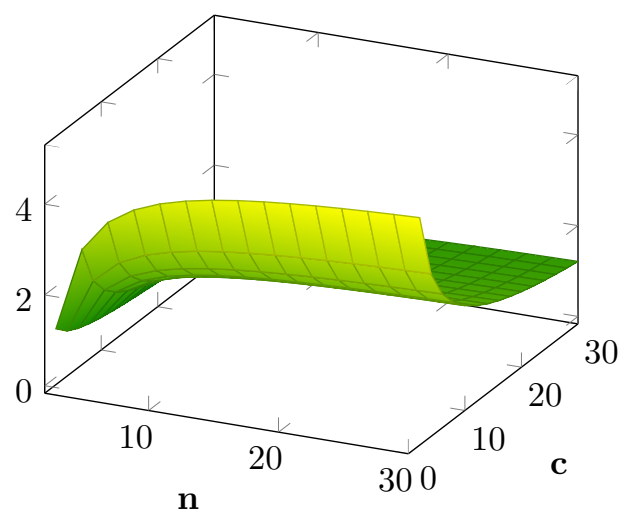

(iv) $\mathrm{m}=1 / 3$

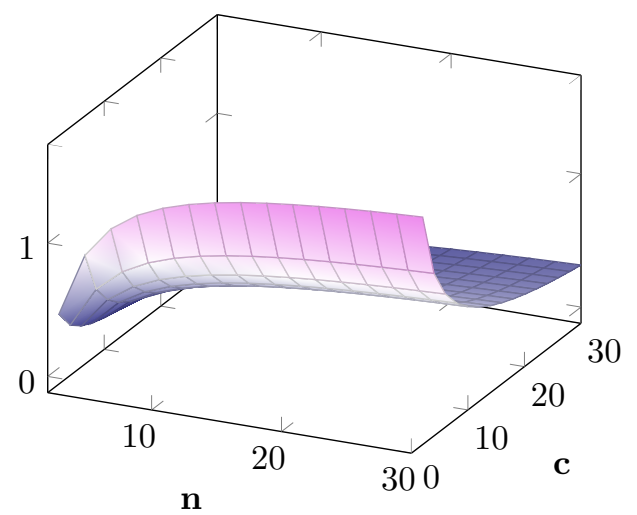

(vi) $\mathrm{m}=3$

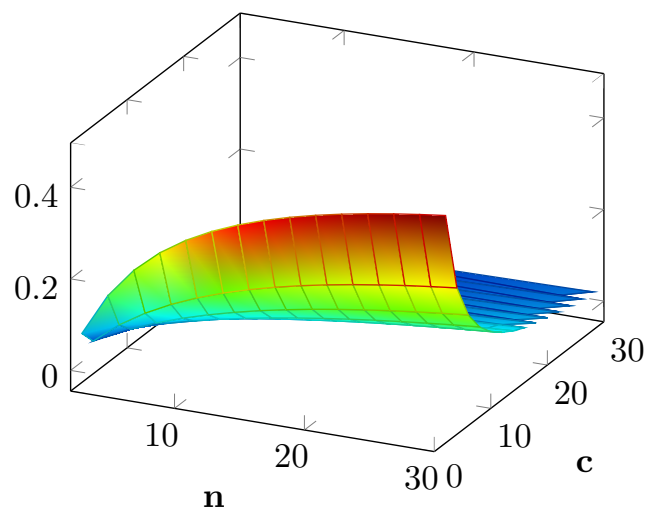

Figure 1. Plots of the coefficient of variation threshold $\mathcal{C V}^{*}(c, m, n)$. 
Specifically, we change the demand slope parameter $m$ in the set $\{1 / 81,1 / 27$, $1 / 9,1 / 3,1,3\}$, and for each value of $m$ we plot the graph of $\mathcal{C V}^{*}$ as a function of $n$ and $c$, when $n$ takes 15 integer values between 2 and 30 and $c$ takes 15 real values between 0.01 and 30.00. These graphs are drawn in Figure 1, illustrating that the threshold value of the coefficient of variation, $\mathcal{C V}^{*}$, is always positive for all considered values of $m, n$, and $c$. Of course, this is not surprising since an observation with $\mathcal{C V}^{*}=0$ would be in contradiction with Klemperer and Meyer (1989) who showed that in the absence of any (demand) uncertainty (i.e., when $\mathcal{C V}=0$ ), the profits from the supply function competition must be always below the profits from the Cournot competition.

Figure 1 also illustrates that an increase in the slope of the demand curve, $m$, reduces the threshold value of the coefficient of variation, $\mathcal{C} V^{*}$, at all values of $n$ and $c$ in their domains. Similarly, the cost parameter $c$ has a negative impact on $\mathcal{C V}^{*}$ at all values of $m$ and $n$ in their domains. On the other hand, the number of firms $n$ is found to have a positive effect on $\mathcal{C V}^{*}$ for all values of $m$ and $c$. As a matter of fact, this effect becomes larger when the cost parameter $c$ is not very high.

\section{Conclusion}

In this paper, we have formalized an insight of Klemperer and Meyer (1989) that the outcome of the supply function competition with demand uncertainty may be more desirable for the firms in the oligopolistic industry than the outcome of the stochastic Cournot competition, and showed how this desirability is affected by some attributes of the industry structure.

In particular, we have found that the higher the slope of the industry demand curve or the higher the cost of producing a unit output or the smaller the number of firms in the industry, the more likely that at any given level of demand uncertainty the supply function competition will bring higher expected profits than the stochastic Cournot competition in equilibrium. We should note that both an increase in the slope of the demand curve and an increase in the production cost of a unit output would result in a decrease in the potential (maximal) social surplus that could be attained in a perfectly competitive industry. Given this fact, our findings suggest that when the potential social surplus in the industry -some part of which the oligopolistic firms can expect to extract when they compete in quantities or in supply functions- is sufficiently small, the

supply function competition with demand uncertainty would become -from the viewpoints of firms- inferior to the stochastic Cournot competition only when 
the size of the demand uncertainty is also sufficiently low. On the other hand, when the number of firms in the industry is not sufficiently small, the supply function competition with demand uncertainty can become a superior mode of competition only at very high levels of uncertainties.

All in all, our findings reveal that whether the Cournot or the supply function competition can yield better outcomes for the oligopolistic firms under demand uncertainty depends on the relative size of this uncertainty (as measured by the

coefficient of variation in demand), with respect to the size of the market and the number of competitors.

\section{References}

Cournot A (1838) Recherches sur les Principes Mathematiques de la Theorie des Richesses. Paris

Delgado J (2006) Coalition-proof supply function equilibria under capacity constraints. Economic Theory 29:219-229

Delgado J, Moreno D (2004) Coalition-proof supply function equilibria in oligopoly. Journal of Economic Theory 114:231-254

Grossman S (1981) Nash equilibrium and the industrial organization of markets with large fixed costs. Econometrica 49:1149-1172

Klemperer PD, Meyer MA (1989) Supply function equilibria in oligopoly under uncertainty. Econometrica 57:1243-1277

Król M (2017) On the equivalence of quantity competition and supply function competition with sunk costs. International Journal Game Theory 46:475-486

Nash J (1950) Equilibrium points in n-person games. Proceedings of the National Academy of Sciences 36:48-49 